\title{
Internal Carotid Artery Blister Aneurysm: Review of Endovascular Techniques
}

\author{
Vipul Gupta ${ }^{1}$ Shrikant Londhe ${ }^{1}$ Rajsrinivas Parthasarathy ${ }^{1}$ \\ ${ }^{1}$ Departement of Interventional Neuroradiology, Artemis Hospital, \\ Address for correspondence Vipul Gupta, MD, Department \\ Gurgaon, Haryana, India \\ of Interventional Neuroradiology, Artemis Hospital, Gurgaon, \\ Haryana 122001, India (e-mail: drvipulgupta25@gmail.com).
}

J Clin Interv Radiol ISVIR 2021;5:158-166.

\begin{abstract}
Cerebral blister aneurysms are very difficult vascular lesions in terms of accurate diagnosis as well as proper management strategy for vascular neurosurgeons and neurointerventionists. Histopathological analysis has revealed their nature to be more akin to pseudoaneurysms in comparison to the typical berry aneurysms. This explains their fragile nature, with high probability of rerupture and regrowth during the follow-up if not addressed properly. Even though surgical approaches have evolved over the years to minimize intraprocedural rupture and recurrence, they still have relatively high morbidity as well as mortality in comparison to endovascular treatment. Over the last few years, endovascular treatment has evolved significantly, with introduction of new devices and techniques. Focus of the endovascular management has shifted from the deconstructive techniques to reconstructive ones with parent artery preservation. Recent introduction of flow diverter technology has enabled neurointerventionists to manage these difficult lesions with more confidence in terms of avoiding rerupture and recurrence. Flow diverter (FD) use in blister aneurysm resulted in high-technical success, complete aneurysm occlusion, and less complications in comparison to other reconstructive techniques like simple coiling, stent assisted coiling, and overlapping/monotherapy stent placement. Few debatable issues like use of dual antiplatelets in the setting of acute subarachnoid hemorrhage (SAH), timing of drugs

\section{Keywords}

- blister aneurysms

- endovascular management

- flow diverter in relation to procedures, single/overlapping devices, and concerns during cerebral vasospasm phase need further detailed analysis and standardized protocols. In this article, we have done a review of different endovascular treatment options in the management of internal carotid artery blister aneurysms with special focus on FD and issues related to it.
\end{abstract}

\section{Introduction}

Intracranial blister aneurysms are uncommon vascular pathology with difficulties encountered in diagnosis as well as management compared with the classical berry aneurysms. Ohara et al reported the first case series of

published online April 14, 2021
DOI https://doi.org/

10.1055s-0041-1726166 ISSN 2457-0214. supraclinoid internal carotid artery (ICA) aneurysm involving its dorsal wall and nonbranching site with extremely fragile nature. They described these aneurysms as wide neck, half dome-shaped and nonbranching site aneurysm with adjacent parent artery involvement. Furthermore, an emphasis was given on their management, as these aneurysms require
(C) 2021. Indian Society of Vascular and Interventional Radiology. This is an open access article published by Thieme under the terms of the Creative Commons Attribution-NonDerivative-NonCommercial-License, permitting copying and reproduction so long as the original work is given appropriate credit. Contents may not be used for commercial purposes, or adapted, remixed, transformed or built upon. (https://creativecommons.org/licenses/by-nc-nd/4.0/). Thieme Medical and Scientific Publishers Pvt. Ltd. A-12, 2nd Floor, Sector 2, Noida-201301 UP, India 
different surgical techniques to exclude them completely from the cerebral circulation compared with traditional one for classical berry aneurysm. ${ }^{1}$ Takahashi used the word "blister" for the first time for these types of aneurysms in 1988 in Japanese literature. ${ }^{2}$

The overall incidence of blister aneurysm is approximately 0.5 to $2 \%$ among all ruptured cerebral aneurysms. ${ }^{1}$ Few case series have kept the term blister aneurysm restricted to only the supraclinoid ICA segment. However, other locations described in English literature are anterior communicating artery (ACOM), basilar artery (BA), and middle cerebral artery (MCA). ${ }^{3,4}$

\section{Pathology}

Histopathological analysis of these aneurysms has been studied extensively and described for the ICA blister location. Ishikawa et al reported blisters as focal arterial wall defect covered with thin fibrous tissue. It is basically platelet plug overlying thin adventitia, which covers defect in intima and media lacking the collagen layer. These features resemble pseudoaneurysms more in comparison to classical berry aneurysms, which show thickened media and adventitia. ${ }^{5}$ This unique morphology of blister aneurysm is responsible for the change in size as well as shape of blister aneurysm in a short period of time. Two basic pathologies have considered to be responsible for blister aneurysm formation, dissection, and atherosclerosis. Focal dissections have been reported, based on angiography and intraoperative findings, but never proved on histopathological analysis. Association of blister aneurysms and atherosclerosis has been proved, based on histopathological analysis. Based on these findings, atherosclerosis and subsequent ulceration can be a factor in the formation of blister aneurysm. ${ }^{6}$ Ogawa et al have suggested association between location of blister aneurysm and systemic hypertension with subsequent increased hemodynamic stress. ${ }^{7}$ Exact pathogenesis is still unclear, but all these associations lead to extremely fragile aneurysms with adjacent parent artery involvement and high tendency to rupture.

\section{Diagnosis}

Considering aggressive natural history of blister aneurysms, diagnosis becomes the key for the early and better management. Computed tomography (CT) and magnetic resonance angiography (MRA) may miss these lesions, owing to their small size, adjacent bony structures, and atypical location. Diagnostic cerebral angiography is gold standard for the diagnosis of blister aneurysm. Typical angiographic features are wide neck, different shapes (fusiform, sessile and saccular) at nonbranching location, adjacent parent artery involvement near the neck of aneurysm, stasis of contrast, and change in morphology over short duration. Changes in aneurysm morphology is considered secondary to organization of blood clot covering the focal arterial wall defect. ${ }^{8}$ These morphological features separate them from the classical berry aneurysm of cerebral circulation. ${ }^{9}$ Digital subtraction angiography (DSA) with three dimensional (3D) rotational angiography helps in correct diagnosis as well as proper treatment planning of blister aneurysm. In the authors' experience, performing DSA in the angles determined using 3D images reveals the true morphology of these aneurysms. In case of initial negative angiogram, early repeat cerebral angiogram is recommended in suspicious cases.

\section{Management}

Considering their extremely fragile wall, lack of definitive neck, small size, and tendency to rerupture as well as regrowth, management of these aneurysms also differs considerably in terms of surgical as well as endovascular approaches. Surgical clipping as a standalone technique has high rates of intraoperative rupture, ischemic complications, and significant recurrence. Gonzalez et al have reported overall intraoperative rupture risk of $28 \%$ for blister aneurysms. ${ }^{10}$ Modifications in surgical approach such as clipping plus wrapping, parallel clipping with involvement of adjacent parent artery, clipping with vascular encircling graft, and trapping with or without bypass have been applied in clinical practice. ${ }^{8,11}$ Peschillo et al in their meta-analysis of 334 patients of ruptured blister aneurysms reported better clinical outcome in endovascular $\operatorname{arm}(78.9 \%)$ in comparison to surgical arm (67.4\%). Combined perioperative morbidity-mortality was more in surgical arm (20 vs. 9\%). ${ }^{9}$ Meta-analysis of surgical management of blister aneurysms has reported surgical morbidity and mortality of approximately $21 \%$ and $17 \%$, respectively. ${ }^{10}$ In a systematic review, clipping alone had highest risk of postoperative aneurysm regrowth (30\%). ${ }^{12}$ In comparison to surgical arm, endovascular approach has significant less morbidity as well as mortality. Ren et al published their retrospective single center data of ruptured blister aneurysm $(n=83)$ managed with microsurgery $(n=33)$ and endovascular $(n=50)$ approaches. Clipping alone (27/33) and stent-assisted coiling (49/50) were most commonly used techniques in microsurgical and endovascular treatment, respectively. They reported more intraoperative aneurysm rupture in the surgical group (14 vs. 4). Favorable clinical outcome was seen in endovascular group in comparison to microsurgery (76 vs. $54 \%$ ). Rebleed rates and retreatment rates were not statistically significant among two groups. ${ }^{12}$ Overall, endovascular group had favorable clinical outcome, less morbidity-mortality, and less incidence of vasospasm-related complications in comparison to the microsurgery group. In meta-analysis of large studies of blister aneurysm treated with endovascular techniques, overall reported morbidity-mortality was 13.4 and $7.3 \%$, respectively. ${ }^{13}$ Recently, endovascular approach has gained popularity in treatment of blister aneurysms, especially after the introduction of flow diverters (FD), which has become the standard method of treatment over the last few years. Overall, endovascular techniques are divided into the deconstructive and reconstructive approach. Deconstructive approach involves parent artery occlusion with or without bypass. Reconstructive approach involves balloon/stent-assisted coiling, single/overlapping stents, covered stent placement, balloon/stent-assisted onyx embolization of the aneurysm sac, and FD stents. ${ }^{13}$ 


\section{Deconstructive Technique}

Early in the endovascular era, parent vessel occlusion was thought to be the most definitive way of treating blister aneurysms to prevent their rerupture and associated morbidity and mortality. Parent vessel occlusion can be done with coils, detachable balloon, and amplatzer plug. Balloon test occlusion has got limitations in the setting of acute subarachnoid hemorrhage (SAH) for assessing collateral flow secondary to vasospasm. ${ }^{14}$ In vasospasm phase of acute subarachnoid hemorrhage, there can be inadequate collateral flow, and patient can develop major ischemic stroke on occluded arterial territory as well as in contralateral circulation secondary to arterial steal phenomenon. In addition, the access to the endovascular treatment for severe vasospasm is limited after parent vessel occlusion. This is a relatively simple procedure for approaching blister aneurysm, but significant risk of major disabling stroke has been reported, especially in the setting of acute SAH. ${ }^{8}$ Rauchaud et al in their meta-analysis reported statistical significant perioperative strokes in deconstructive approach compared with the reconstructive approach (29\% vs. 5\%). ${ }^{13}$ Hence, reconstructive techniques have been considered more favorable for better clinical outcome.

\section{Reconstructive Technique}

Reconstructive technique mainly aims to exclude the aneurysm from cerebral circulation by maintaining parent artery blood flow.

\section{Aneurysm Coiling}

Small size of the aneurysm sac and extremely fragile dome makes the aneurysm sac catheterization extremely difficult with high risk of intraoperative rupture. Wide neck of the aneurysm poses significant doubts about coil mass stability inside the aneurysm sac. Coil prolapse and thromboembolic complications are very high in these cases. Dynamic nature of the underlying disease can result in high chance of aneurysm regrowth and rerupture in future. Park et al have reported regrowth of the aneurysm in all four cases of endovascular primary coil embolization of blister aneurysm with two of them rebleed. ${ }^{15}$ Overall, simple/balloon-assisted coiling has high risk of intraoperative rupture, thromboembolic complications secondary to coil prolapse, recurrence, and rerupture. In fact, balloon inflation can be potentially hazardous in blister aneurysm, as underlying parent vessel near the neck is mostly involved in blister aneurysms.

\section{Stent (Monotherapy or Overlapping)/Stent-Assisted Coiling}

Stent-assisted coiling can provide potential advantages of coiling (leading to immediate aneurysm occlusion) and stenting (parent artery reconstruction to avoid recurrence). However, it is technically challenging and increases complexity of the procedure. Multiple case series have reported the use of stent-assisted coiling, overlapping stents, and stent monotherapy for the treatment of blister aneurysm. ${ }^{11,16,17}$
Stent monotherapy or telescoping stent treatment options in blister aneurysm was reported in a few case series. The rationale for using stent without additional coils was to decrease the intra-aneurysmal blood flow and wall shear stress with progressive endothelization, leading to complete exclusion of aneurysm (-Fig. 1). This avoids catheterization of aneurysm sac for coiling and hence probably avoiding fatal intraoperative rupture. Walsh et al in their case series $(n=8)$ of stenting without additional coiling showed $75 \%$ complete or stable aneurysm occlusion with two patients (25\%) requiring retreatment in the form of parent vessel occlusion. They used overlapping stent in seven patients and one patient was treated with stent monotherapy. Good clinical outcome was noted in all patients without any rerupture. They suggested that stent monotherapy or telescoping stent technique (preferably) is safe and feasible technique in the management of blister aneurysms. ${ }^{11}$

Stent-assisted coiling has theoretical advantage of immediate aneurysm sac protection in comparison to only stent approach. Meckel et al in their case of 11 patients of ruptured blister aneurysm treated with stent-assisted coiling using Neuroform stent (Stryker Neurovascular, California) showed complete/stable angiographic occlusion of the aneurysm during follow-up in 10 cases. One patient had asymptomatic rebleed and was treated with parent vessel occlusion. Two patients had recurrent aneurysm growth at 1-month follow-up angiogram which was treated with parent artery


Fig. 1 A 58-year-old male patient presented with acute subarachnoid hemorrhage (SAH). 3D rotational angiographic solid-state drive (SSD) image and corresponding 2D digital subtraction angiography (DSA) image showed classical blister aneurysm of supraclinoid internal carotid artery (ICA) (arrows in $\mathbf{A}$ and $\mathbf{B}$ ). This patient was treated with two overlapping stents (Enterprise-Codman Neuro) across the blister aneurysm (arrow. in C). Six-month follow-up DSA images showed complete aneurysm occlusion (D). 
occlusion in one and coiling in another patient. Good clinical outcome was seen in 10/11 patients. They concluded that stent-assisted coiling is a viable option but may need early follow-up angiogram and retreatment if necessary. ${ }^{17}$

Closed cell design stents may provide potential advantage of resheath and reposition of the device in comparison to the open cell design stent in addition to coil mass support. Xu et al in their study ( $n=44)$ of ruptured blister aneurysms, 9 were treated with single stent-assisted coiling and 35 with telescoping stents using closed cell design Enterprise (Cordis Neurovascular). Two patients in the single-stent group had recurrence, which was addressed by using second stent. The patient treated with telescoping stents had better immediate complete aneurysm occlusion as compared with single stent. ${ }^{18}$ These case series have highlighted the importance of the multiple overlapping stents with the intention of additional FD effect. Another study has reported good clinical outcome in $14 / 17$ patients in their single center experience of 17 ruptured blister aneurysms. A total of six patients were treated with single stent (Enterprise), five with overlapping stents, and six with single stent assisted coiling. Among single stent patient subgroup, two patients (2/6) had aneurysm regrowth, which were treated with additional stent placement at the time of early angiogram (10-14 days). One interesting finding in their study was high probability of arterial dissection in the patients of blister aneurysm during diagnostic angiogram and therapeutic procedure (3/17), raising the possibility of underlying connective tissue disorder. Complete aneurysm occlusion was noted in 11/14, incomplete stable occlusion in $1 / 14$, and retreatment in $2 / 14 .^{19}$ Their result showed that overlapping stent group had better aneurysm occlusion with less rates of retreatment.

Gonzalez et al in their systematic review have reported combined morbidity-mortality of $10 \%$ and $8 \%$ for stent-assisted coiling and overlapping stent, respectively. The rate of regrowth (38\%) and rebleed (12.5\%) was higher in the stenting alone subgroup. They further suggested that only stenting may not always provide immediate aneurysm protection, considering the aggressive natural history of blister aneurysms. ${ }^{10}$ One of the authors' experience also showed incomplete aneurysm occlusion (30\%) in only stent/overlapping stents without coiling, exposing to the risk of repeat hemorrhage. ${ }^{19}$

\section{Flow Diverter (FD)}

FD stents are basically braided stents with high-pore density and low porosity. Deployed FD modifies intra-aneurysmal blood flow to decrease wall shear stress along with maintaining laminar blood flow in the parent artery. Computation flow dynamics study in the aneurysm treated with FD placement has showed significantly decreased intra-aneurysm flow velocity, flow volume, and wall shear stress (WSS). ${ }^{20}$ Progressive thrombosis of the aneurysm and endothelization of the stent result in healing of the aneurysm neck with complete exclusion from the circulation.

FD use in blister aneurysms was considered in clinical practice, as it leads to decreased flow into the aneurysm sac with subsequent endothelization, preserved flow in covered side branches, and no need for the aneurysm catheterization for adjuvant coiling. However, issues like lack of immediate aneurysm sac exclusion from circulation and probable long waiting time for complete occlusion do pose challenges for their practical consideration in blister aneurysms. Another important aspect of FD treatment in ruptured cerebral aneurysms is proper antiplatelet regimen, its timing, as well as dose modification in relation to other surgical interventions like external ventricular drain (EVD)/ventriculoperitoneal (VP) shunt/tracheostomy/decompressive craniectomy.

Recently, a few case series have reported safety and efficacy of the FD in treatment of blister aneurysm using pipeline embolization device (PED, Medtronic). Ryan et al in their series of 13 ruptured blister aneurysms patients showed good clinical outcome in $77 \%$ (10/13) and combined morbidity-mortality in three patients. There was one intraprocedural complication of wire-related perforation during the delivery of second overlapping FD, leading to significant morbidity and requiring decompressive craniectomy. In their case series, only two patients were treated using telescoping FD stents. As much as 50\% patients (5/10) had complete aneurysm occlusion on 3 months follow-up angiogram and remaining at 12 months. Two patients, who had residual filling or increased remnant of the aneurysm during follow-up, were treated with another FD. No patient had procedural or delayed aneurysmal rebleeding. They concluded that FD is safe and effective in the treatment of blister aneurysms, especially in good clinical grade patients (Hunt and Hess Grade of I-III). ${ }^{21}$

Yoon et al in their multicenter case series of 11 patients showed $83 \%$ good clinical outcome with no procedural or post-FD aneurysm rerupture. Significant morbidity-mortality was reported in three patients secondary to vasospasm, thromboembolic complications, and monocular blindness secondary to covered ophthalmic artery decreased flow after telescoping FD placement. One patient had asymptomatic complete stent thrombosis with ICA occlusion during follow-up. In this series, most patients were treated with single FD (7/11) with complete aneurysm occlusion during follow-up. ${ }^{22}$

Mokin et al in their multicenter retrospective study of 43 patients with ICA blister aneurysm treated with PED reported complete aneurysm occlusion in $88 \%$ of patients on 4 months follow-up angiogram and rest with either reduced filling or residual filling. One patient has aneurysm rerupture on day 4 of the treatment, which might suggest toward difference of blood pressure targets in blister aneurysm compared with another aneurysm treated with FD. Thromboembolic complications were noted in 5/43 patients with intraprocedural rupture in one patient. The use of adjuvant coiling did not affect the aneurysm occlusion during follow-up angiogram. Another important observation was lack of contrast stasis in blister aneurysm post deployment of FD, which is not predictor of complete aneurysm occlusion during follow-up angiogram. Overall, FD treatment is safe and effective in ruptured blister aneurysms. ${ }^{23}$

Meta-analysis of all 62 ruptured blister aneurysms treated with FD showed complete occlusion in 91\% mid to long-term follow-up. Compared with other reconstructive techniques, FD group showed more complete occlusion 
Table 1 Different studies of FD experience in blister aneurysm

\begin{tabular}{|c|c|c|c|c|c|}
\hline Study & $\begin{array}{l}\text { No. of } \\
\text { patients }\end{array}$ & Angiographic outcome & $\begin{array}{l}\text { Clinical outcome } \\
(\mathrm{mRS}<2)\end{array}$ & $\begin{array}{l}\text { Procedure-related } \\
\text { complications }\end{array}$ & Remarks \\
\hline Chalouhi et al ${ }^{27}$ & 8 & $\begin{array}{l}\text { Complete occlusion (5/6), } \\
\text { partial filling in one }\end{array}$ & All & None & $\begin{array}{l}\text { Seven aneurysms were located } \\
\text { at ICA and one at basilar artery }\end{array}$ \\
\hline Hu et al ${ }^{41}$ & 3 & Complete occlusion (3/3) & All & None & $\begin{array}{l}\text { All the aneurysms were located } \\
\text { at ICA }\end{array}$ \\
\hline Lin et al ${ }^{42}$ & 7 & Complete occlusion (7/7) & All & None & \\
\hline Linfante et al ${ }^{43}$ & 10 & $\begin{array}{l}\text { Complete occlusion } \\
(9 / 10)\end{array}$ & $\begin{array}{l}\text { 9/10 } \\
\text { One mortality sec- } \\
\text { ondary to severe } \\
\text { vasospasm }\end{array}$ & None & $\begin{array}{l}8 / 10 \text { aneurysms were located } \\
\text { at ICA, } 2 / 10 \text { were located at } \\
\text { MCA. } \\
\text { All were treated with single } \\
\text { device. }\end{array}$ \\
\hline Ryan et $a^{21}$ & 13 & $\begin{array}{l}\text { Complete occlusion in } \\
5 / 11 \text {, reduced filling in } \\
\text { two and retreatment in } \\
\text { two patients. }\end{array}$ & $\begin{array}{l}10 / 11 \\
\text { mRS } 4 \text { in one } \\
\text { patient }\end{array}$ & $\begin{array}{l}\text { 1. Wire perforation lead- } \\
\text { ing to ICH. } \\
\text { 2. Worsening IVH leading } \\
\text { to death } \\
\text { 3. Rupture of another } \\
\text { aneurysm leading to } \\
\text { death }\end{array}$ & $\begin{array}{l}11 \text { patients received one device } \\
\text { and two patients were } \\
\text { treated with telescoping } \\
\text { device (ICA) }\end{array}$ \\
\hline Yoon et al22 & 11 & $\begin{array}{l}\text { Complete occlusion } \\
(7 / 11) \\
\text { Partial filling in one. }\end{array}$ & $9 / 11$ & $\begin{array}{l}\text { 1. Thromboembolic } \\
\text { 2. Vision loss } \\
\text { 3. ICH resulting in death }\end{array}$ & $\begin{array}{l}\text { During follow up one patient } \\
\text { had asymptomatic in-stent } \\
\text { stenosis (ICA) }\end{array}$ \\
\hline Mokin et $\mathrm{al}^{23}$ & 43 & $\begin{array}{l}\text { Complete occlusion in } \\
87 \%(28 / 32) \text {, three with } \\
\text { reduced filling and one } \\
\text { with persistent filling of } \\
\text { the aneurysm }\end{array}$ & $26 / 38(68 \%)$ & $\begin{array}{l}\text { 1. Thromboembolic in } \\
\text { five patients. } \\
\text { 2. Intraprocedural rup- } \\
\text { ture in one patient. } \\
\text { 3. Worsening of IVH in } \\
\text { one patient. } \\
\text { 4. Rerupture of aneurysm } \\
\text { in one patient on day } 4 \\
\text { of treatment. }\end{array}$ & $\begin{array}{l}\text { All the aneurysms were located } \\
\text { at ICA }\end{array}$ \\
\hline
\end{tabular}

Abbreviations: FD, flow diverter; ICA, internal carotid artery; ICH, intracerebral hemorrhage; IVH, intraventricular hemorrhage; mRS, modified Rankin score.

(91 vs. 68\%) and lower rates of retreatment (6.6 vs. $30.7 \%)$. No significant difference was noted in early rebleeding; however, procedural complications and perioperative stroke were noted in FD group compared with other reconstructive techniques. ${ }^{13}$ We further discuss the technical issues and antiplatelet dosage dilemma during FD treatment of blister aneurysm (-Table 1).

\section{Single versus Overlapping FD}

Although overlapping FD might appear to be beneficial approach in achieving high degree of flow diversion, it has its own limitation in terms of more thromboembolic complications and technical complexity. ${ }^{24}$ Xiang et al in their in vitro study showed that single device strategy of the FD might work for the treatment of the blister aneurysm. Single FD with compaction of device near the aneurysm neck or inflow will significantly reduce the intra-aneurysm blood flow velocity as well as wall WSS. ${ }^{20}$

Overlapping FD placement increases the risk of thromboembolic complications, technical complexity, and more risk of side branch occlusion and in-stent stenosis. ${ }^{25}$ One of the authors has reported good clinical outcome with a single FD device in blister aneurysms, with compaction near the neck of the aneurysm and good wall apposition (-Fig. 2). ${ }^{26}$


Fig. 2 A 34-year-old male patient presented with acute subarachnoid hemorrhage (SAH) (Grade III) on CT brain (A). 3D rotational solid-state drive (SSD) image showed broad neck small aneurysm along dorsomedial wall of supraclinoid internal carotid artery (ICA), the typical site of blister aneurysm (arrow in $\mathbf{B}$ and $\mathbf{C}$ ). Since patient was drowsy (Glasgow coma scale [GCS]-E3V4M5), external ventricular drain (EVD) placement was done prior to procedure. Repeat CT showed EVD in place (arrow in D) with no fresh bleed, following which antiplatelet loading dose was done. After pipeline embolization device (PED) placement, digital subtraction angiography (DSA) image showed aneurysmal filling with stasis of contrast in the aneurysm in delayed phase (arrow in $\mathbf{F}$ only). Vaso-CT image showed stent compaction near the neck of the aneurysm with good wall opposition of the device (arrow in G). 6-month follow-up DSA showed complete aneurysm occlusion with preserved flow in covered side branches $(\mathbf{H})$. 


\section{Antiplatelets}

One of the most important issues for FD use in ruptured blister aneurysms is antiplatelet regimen selection and their proper timing in relation to the procedure. The best protocol is to have antiplatelet drugs which have maximum activity in circulatory system close to time of FD deployment in order to avoid thromboembolic complications as well as potentially avoiding rebleed before the treatment. Although few case series have highlighted the use of FD after the acute phase of SAH to avoid antiplatelet related issues, deferring the treatment especially in case of blister aneurysm is debatable, considering their aggressive natural history. ${ }^{27}$ Most reported case series of FD in blister aneurysm have reported antiplatelet protocol of aspirin $(650 \mathrm{mg})$ and clopidogrel $(600 \mathrm{mg})$ at least 6 to 8 hours prior to treatment, considering the maximal platelet inhibition of these two drugs. ${ }^{21,27,28}$ Another case series used intravenous aspirin (500 $\mathrm{mg}$ ) just prior to FD deployment and oral clopidogrel (600 mg) after the procedure. ${ }^{25}$ Intravenous aspirin is not available in India and resistance to clopidogrel is well-known from the cardiac literature ranging from 5 to $44 \%{ }^{29}$ Platelet function assays have shown significant variation in terms of results and predictability. All these issues have led to consideration of newer antiplatelet agents in the endovascular procedures. Prasugrel is thienopyridine prodrug, which is less likely to get affected by genetic polymorphism of cytochrome enzymes in comparison to clopidogrel. In addition to this, it achieves faster and higher platelet aggregation inhibition in comparison to clopidogrel..$^{30}$ Another new antiplatelet agent, ticagrelor, is a reversible direct inhibitor of the P2Y12 ADP receptors. Ticagrelor does not need metabolic conversion and has faster onset of action than clopidogrel. ${ }^{31}$

The author has reported use of loading dose of prasugrel (50 mg loading dose followed by $10 \mathrm{mg}$ maintenance) and aspirin (300 mg loading followed by $150 \mathrm{mg}$ maintenance) in nine patients with no rebleed, ischemic complications, or in-stent thrombus formation. ${ }^{26}$ The protocol was to time the drug administration 2 hours prior to FD deployment. The other option is to use ticagrelor (180 $\mathrm{mg}$ loading dose followed by $90 \mathrm{mg}$ maintenance), particularly in patients with recent transient ischemic attacks/stroke (clinical and or imaging) and old age (age more than 75 years). ${ }^{31}$ Recent meta-analysis has reported reduced thromboembolic complications with newer antiplatelets (ticagrelor and prasugrel) in comparison to clopidogrel without higher risk of hemorrhagic complications. They concluded that both ticagrelor and prasugrel are safe and effective alternative to clopidogrel for patients treated with FD, especially in hyporesponders/nonresponders. ${ }^{32}$ Most of the case series had reported protocol of dual antiplatelets for 3 to 6 months followed by aspirin monotherapy till 1 year. ${ }^{27,28}$ In the author's institution, dual antiplatelets are given for a 1-year period and aspirin monotherapy continues lifelong.

Another option for antiplatelet regimen is to use intravenous GPIIb/IIIa inhibitors in the setting of FD placement for ruptured blister aneurysms. Limaye et al have used IV tirofiban infusion just before deployment of FD/stent followed by oral antiplatelets loading for the patients requiring EVD.
They used $0.10 \mathrm{ug} / \mathrm{kg} / \mathrm{min}$ IV tirofiban infusion as monotherapy without any loading dose followed by dual antiplatelet loading postprocedure in 19 patients. In their series, two patients had asymptomatic EVD tract-related hemorrhages. One patient had transient weakness after stopping the tirofiban infusion after antiplatelet loading. One patient had significant thrombocytopenia which was reversible within 24 hours after discontinuation of tirofiban infusion. They concluded that IV tirofiban infusion as monotherapy is safe and effective whenever stent or FD use is anticipated in aneurysm treatment. ${ }^{33}$

One of the challenges in using dual antiplatelets in acutely ruptured aneurysms is surgical interventions in the form of EVD change/removal, VP shunt placement, decompressive craniectomy (DC), and tracheostomy, which may be needed in these patients ( - Fig. 2). Systemic meta-analysis has reported 3 to $10 \%$ EVD-related radiographic hemorrhagic complications in the patients on dual antiplatelets in comparison to $0.9 \%$ in convention coiling group. ${ }^{34}$ Recent retrospective single center analysis has showed no clinically significant hemorrhagic complications after EVD placement/EVD change/VP shunt placement even through radiological hemorrhages were more in patients receiving dual antiplatelets (9 vs. 1 patient). ${ }^{35,36}$ At the author's institution, the following approach is followed in these circumstances for any SAH patient on dual antiplatelets requiring additional surgical interventions:

1) In acute SAH with hydrocephalus, EVD is placed prior to procedure with controlled drainage. We repeat CT brain 2 hours after EVD placement to confirm tube position and assess for tract hemorrhage prior to antiplatelet loading dose administration.

2 ) In case of a patient requiring EVD change, one may have to time if possible such that 4 to 5 half-lives (at least 2 half-lives) have elapsed from the last dose of ticagrelor or prasugrel. This is to ensure the transfused platelets are functionally active in vivo to prevent hemorrhage during this process.

\section{FD and Cerebral Vasospasm}

Cerebral vasospasm following acute SAH poses unique challenge for FD placement in terms of proper sizing of device and wall apposition for adequate flow diversion. The author performs gradual intra-arterial nimodipine infusion prior to sizing of the device as well as deployment. Another issue is the risk of FD retraction during vasospasm period, leading to loss of aneurysm neck coverage and rerupture. One case report has mentioned this phenomenon probably secondary to ICA vasospasm at the time of FD placement with subsequent dilatation of vessel and rerupture on day five, leading to retreatment in form of parent vessel occlusion with external carotid artery (ECA)-MCA bypass. ${ }^{37}$ In the author's experience, selection of proper distal landing zone and adequate distal anchoring of flow diverter is recommended to avoid foreshortening of the device. This also enables dynamic push-pull maneuver to achieve the device compaction near the neck of the aneurysm. Since there is risk of reduced flow in covered side branches, sometimes this can be detrimental in patients of cerebral vasospasm following acute SAH ( - Fig. 3 ). 

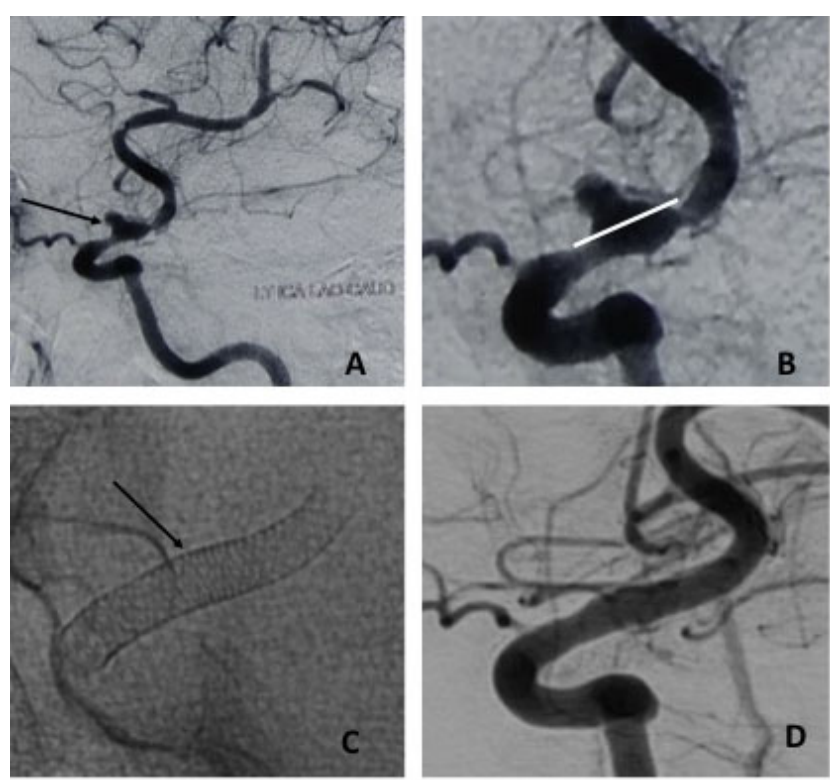

Fig. 3 A 50-year-old female patient presented with acute subarachnoid hemorrhage $(\mathrm{SAH})$. Cerebral angiogram showed the aneurysm in dorsal supraclinoid internal carotid artery (ICA)-typical location for blister aneurysms (arrow in A). Distal intracranial arteries showed diffuse vasospasm. Magnified image of blister aneurysm with adjacent parent artery involvement (white line indication neck of the aneurysm in B). Flow diverter (FD) (Pipeline. Medtronic) placement for the treatment of blister aneurysm with stent compaction near the aneurysmal segment of ICA (arrow in C). The FD expansion has remodeled the artery in the areas of spasm. This has ensured proper apposition to the arterial wall. Six months follow-up angiogram showed complete aneurysm occlusion with parent artery reconstruction (D). (Reprinted with permission from Indian journal of neurosurgery from the review article. "Endovascular Management of Cerebral Aneurysm: The Recent Trends" by Gupta et al. August 2018).

Medical management of cerebral vasospasm in the form of aggressive hypertension is still unclear, especially in cases of persistent aneurysm filling after FD.

\section{Recent Advances in FD Technology}

Pipeline shield technology-Surface coated PED shield technology is basically phosphorylcholine coating of $3 \mathrm{~nm}$ along the braids of FD. This coating reduces platelet adhesion and subsequent aggregation to the FD surface, reducing thromboembolic complications. In vitro studies using this shield technology have shown early stent endothelialization compared to PED. Recently, one single center case series of 14 patients of ruptured aneurysm treated with this technology has shown good clinical outcome using single antiplatelet aspirin. Two aneurysms in their series were blister aneurysm with complete occlusion in follow-up. ${ }^{38}$ They used aspirin loading dose with intra-operative bridging of abciximab in a few patients. Five patients were started on postoperative heparin infusion in addition to aspirin, two of whom had rebleeding from aneurysm. They concluded that in addition to aspirin, no other antiplatelets/anticoagulation therapy is necessary for pipeline shield for prevention of ischemic complications. Trivelato et al also reported safe use of pipeline shield in blister aneurysm with complete aneurysm occlusion. ${ }^{39}$

\section{Other Approaches \\ Covered Stents}

Even though covered stents are used routinely for cervical ICA aneurysms/pseudoaneurysms, intracranial application of these devices is limited. The most common reasons are their flexibility and navigation issues in tortuous anatomy of intracranial ICA. Recent development in guide catheters can solve these issues by their optimal support for stent navigation as well as stent delivery. Willis covered stent is a balloon expandable device, specifically designed for the intracranial vasculature. Fang et al have reported use of the Willis covered stent in 11 patients of ruptured ICA blister aneurysm with good clinical outcome in all patients. Complete aneurysm occlusion was noted in all the patients in follow-up angiograms. Ophthalmic artery in one and anterior choroidal artery in another patient was covered during stent placement, but none developed clinical symptoms. Two patient had in-stent stenosis secondary to neointimal hyperplasia for which dual antiplatelet were continued. ${ }^{40}$ Even though initial results are promising, device availability, longer follow-up, and fate of covered side branches, especially anterior choroidal artery, are issues that remain and make the practical consideration of this treatment selection debatable, especially in the current FD era.

\section{Balloon/Stent Assisted Onyx Embolization}

Ashour et al have reported intrasaccular blister aneurysm embolization using Onyx HD 500 with balloon and/or stent assistance. In this technique, slow injection of the onyx was done in view to secure the aneurysm sac with inflated balloon. If needed, this was followed by the stent placement through another microcatheter to avoid onyx cast migration. ${ }^{8}$ Operator's comfort and experience is of utmost important to prevent thromboembolic complications. Onyx has the advantage of forming immediate solid cast confirming the aneurysm morphology and least possibility of aneurysm sac rupture during the procedure in comparison to coils. However, there are very few reports and in view of weakened vessel wall, the aneurysm may keep growing with this approach. In the era of FD, this approach may not be practical in the management of blister aneurysm.

\section{Conclusion}

Blister aneurysm management has always been challenging to neurointerventionists as well as neurosurgeons. In comparison to surgical arm, endovascular therapy has showed good outcome with less morbidity and mortality. Technical innovations have shifted endovascular techniques focus from deconstructive to reconstructive after the introduction of stents. The recent introduction of FD technology has revolutionized the management of blister aneurysms. Still, addressing certain issues like dual antiplatelet dosages, their timing prior to FD, and technical difficulty during vasospasm period may need further research for standardized protocols. In future, coated FD like pipeline shield technology and mini-FD may play an increasing role in the treatment of blister aneurysms. 


\section{Conflict of Interest}

None declared.

\section{References}

1 Abe M, Tabuchi K, Yokoyama H, Uchino A. Blood blisterlike aneurysms of the internal carotid artery. J Neurosurg 1998;89(3):419-424

2 Regelsberger J, Matschke J, Grzyska U, et al. Blister-like aneurysms-a diagnostic and therapeutic challenge. Neurosurg Rev 2011;34(4):409-416

3 Cıkla U, Sadighi A, Bauer A, Başkaya MK. Fatal ruptured blood blister-like aneurysm of middle cerebral artery associated with Ehlers-Danlos syndrome type VIII (periodontitis type). J Neurol Surg Rep 2014;75(2):e210-e213

4 Andaluz N, Zuccarello M. Blister-like aneurysms of the anterior communicating artery: a retrospective review of diagnosis and treatment in five patients. Neurosurgery 2008;62(4):807811, discussion 811

5 Ishikawa T, Nakamura N, Houkin K, Nomura M. Pathological consideration of a "blister-like" aneurysm at the superior wall of the internal carotid artery: case report. Neurosurgery 1997;40(2):403-405, discussion 405-406

6 Peitz GW, Sy CA, Grandhi R. Endovascular treatment of blister aneurysms. Neurosurg Focus 2017;42(6):E12

7 Ogawa A, Suzuki M, Ogasawara K. Aneurysms at nonbranching sites in the surpaclinoid portion of the internal carotid artery: internal carotid artery trunk aneurysms. Neurosurgery 2000;47(3):578-583, discussion 583-586

8 Ashour R, Dodson S, Aziz-Sultan MA. Endovascular management of intracranial blister aneurysms: spectrum and limitations of contemporary techniques. J Neurointerv Surg 2016;8(1):30-37

9 Peschillo S, Cannizzaro D, Caporlingua A, Missori P. A systematic review and meta-analysis of treatment and outcome of blister-like aneurysms. AJNR Am J Neuroradiol 2016;37(5):856-861

10 Gonzalez AM, Narata AP, Yilmaz H, et al. Blood blister-like aneurysms: single center experience and systematic literature review. Eur J Radiol 2014;83(1):197-205

11 Walsh KM, Moskowitz SI, Hui FK, Spiotta AM. Multiple overlapping stents as monotherapy in the treatment of 'blister' pseudoaneurysms arising from the supraclinoid internal carotid artery: a single institution series and review of the literature. J Neurointerv Surg 2014;6(3):184-194

12 Ren Y, Liu L, Sun H, et al. Microsurgical versus endovascular treatments for blood-blister aneurysms of the internal carotid artery: a retrospective study of 83 patients in a single center. World Neurosurg 2018;109:e615-e624

13 Rouchaud A, Brinjikji W, Cloft HJ, Kallmes DF. Endovascular treatment of ruptured blister-like aneurysms: a systematic review and meta-analysis with focus on deconstructive versus reconstructive and flow-diverter treatments. AJNR Am J Neuroradiol 2015;36(12):2331-2339

14 Sorteberg A. Balloon occlusion tests and therapeutic vessel occlusions revisited: when, when not, and how. AJNR Am J Neuroradiol 2014;35(5):862-865

15 Park JH, Park IS, Han DH, et al. Endovascular treatment of blood blister-like aneurysms of the internal carotid artery. J Neurosurg 2007;106(5):812-819

16 Ahn JY, Cho JH, Jung JY, Lee BH, Yoon PH. Blister-like aneurysms of the supraclinoid internal carotid artery: challenging endovascular treatment with stent-assisted coiling. J Clin Neurosci 2008;15(9):1058-1061

17 Meckel S, Singh TP, Undrén P, et al. Endovascular treatment using predominantly stent-assisted coil embolization and antiplatelet and anticoagulation management of ruptured blood blister-like aneurysms. Am J Neuroradiol 2011; 32(4):764-771

18 Xu D, Zhang C, Wang T, et al. Evaluation of enterprise stent-assisted coiling and telescoping stent technique as treatment of supraclinoid blister aneurysms of the internal carotid artery. World Neurosurg 2018;110:e890-e896

19 Chinchure SD, Gupta V, Goel G, Gupta A, Jha A. Subarachnoid hemorrhage with blister aneurysms: Endovascular management. Neurol India 2014;62(4):393-399

20 Xiang J, Damiano RJ, Lin N, et al. High-fidelity virtual stenting: modeling of flow diverter deployment for hemodynamic characterization of complex intracranial aneurysms. J Neurosurg 2015;123(4):832-840

21 Ryan RW, Khan AS, Barco R, Choulakian A. Pipeline flow diversion of ruptured blister aneurysms of the supraclinoid carotid artery using a single-device strategy. Neurosurg Focus 2017;42(6):E11

22 Yoon JW, Siddiqui AH, Dumont TM, et al. Endovascular Neurosurgery Research Group. Feasibility and safety of pipeline embolization device in patients with ruptured carotid blister aneurysms. Neurosurgery 2014;75(4):419-429, discussion 429

23 Mokin M, Chinea A, Primiani CT, et al. Treatment of blood blister aneurysms of the internal carotid artery with flow diversion. J Neurointerv Surg 2018;10(11):1074-1078

24 Damiano RJ, Tutino VM, Paliwal N, et al. Compacting a single flow diverter vs. overlapping flow diverters for intracranial aneurysm: a computational study. Am J Neuroradiol 2017; 38(3):603-610

25 Yang C, Vadasz A, Szikora I. Treatment of ruptured blood blister aneurysms using primary flow-diverter stenting with considerations for adjunctive coiling: a single-centre experience and literature review. Interv Neuroradiol 2017;23(5):465-476

26 Parthasarathy R, Gupta V, Gupta A. Safety of prasugrel loading in ruptured blister like aneurysm treated with a Pipeline device. Br J Radiol 2018;91(1086):20170476

27 Chalouhi N, Zanaty M, Tjoumakaris S, et al. Treatment of blister-like aneurysms with the pipeline embolization device. Neurosurgery 2014;74(5):527-532, discussion 532

28 Cerejo R, Bain M, John S, et al. Flow diverter treatment of cerebral blister aneurysms. Neuroradiology 2017;59(12):1285-1290

29 Ray S. Clopidogrel resistance: the way forward. Indian Heart J 2014;66(5):530-534

30 Mega JL, Close SL, Wiviott SD, et al. Cytochrome P450 genetic polymorphisms and the response to prasugrel: relationship to pharmacokinetic, pharmacodynamic, and clinical outcomes. Circulation 2009;119(19):2553-2560

31 Clemmensen P, Dridi NP, Holmvang L. Dual antiplatelet therapy with prasugrel or ticagrelor versus clopidogrel in interventional cardiology. Cardiovasc Drugs Ther 2013;27(3):239-245

32 Podlasek A, Al Sultan AA, Assis Z, Kashani N, Goyal M, Almekhlafi MA. Outcome of intracranial flow diversion according to the antiplatelet regimen used: a systematic review and meta-analysis. J Neurointerv Surg 2020;12(2):148-155

33 Limaye K, Zanaty M, Hudson J, et al. The safety and efficacy of continuous tirofiban as a monoantiplatelet therapy in the management of ruptured aneurysms treated using stent-assisted coiling or flow diversion and requiring ventricular drainage. Neurosurgery 2019;85(6):E1037-E1042

34 Akbari SH, Reynolds MR, Kadkhodayan Y. Cross DT III, Moran CJ. Hemorrhagic complications after prasugrel (Effient) therapy for vascular neurointerventional procedures. J Neurointerv Surg 2013;5(4):337-343

35 Bodily KD, Cloft HJ, Lanzino G, Fiorella DJ, White PM, Kallmes DF. Stent-assisted coiling in acutely ruptured 
intracranial aneurysms: a qualitative, systematic review of the literature. Am J Neuroradiol 2011;32(7):1232-1236

36 Hudson JS, Prout BS, Nagahama Y, et al. External ventricular drain and hemorrhage in aneurysmal subarachnoid hemorrhage patients on dual antiplatelet therapy: a retrospective cohort study. Neurosurgery 2019;84(2):479-484

37 Heit JJ, Telischak NA, Do HM, Dodd RL, Steinberg GK, Marks MP. Pipeline embolization device retraction and foreshortening after internal carotid artery blister aneurysm treatment. Interv Neuroradiol 2017;23(6):614-619

38 Manning NW, Cheung A, Phillips TJ, WenderothJD. Pipeline shield with single antiplatelet therapy in aneurysmal subarachnoid haemorrhage: multicentre experience. J NeuroInterventional Surg 2019;11(7):694-698

39 Trivelato FP, Wajnberg E, Rezende MTS, et al. Safety and effectiveness of the pipeline flex embolization device with shield technology for the treatment of intracranial aneurysms: midterm results from a multicenter study. Neurosurgery 2020;87(1):104-111

40 Fang C, Tan H-Q Han H-J, et al. Endovascular isolation of intracranial blood blister-like aneurysms with Willis covered stent. J Neurointerv Surg 2017;9(10):963-968

41 Hu YC, Chugh C, Mehta H, Stiefel MF. Early angiographic occlusion of ruptured blister aneurysms of the internal carotid artery using the Pipeline Embolization Device as a primary treatment option. J Neurointerv Surg 2014;6(10):740-743

42 Lin N, Brouillard AM, Keigher KM, et al. Utilization of pipeline embolization device for treatment of ruptured intracranial aneurysms: US multicenter experience. J Neurointerv Surg 2015;7(11):808-815

43 Linfante I, Mayich M, Sonig A, Fujimoto J, Siddiqui A, Dabus G. Flow diversion with pipeline embolic device as treatment of subarachnoid hemorrhage secondary to blister aneurysms: dual-center experience and review of the literature. J Neurointerv Surg 2017;9(1):29-33 